\title{
Animal model for chronic massive rotator cuff tear: behavioural and histologic analysis
}

\author{
N. Sevivas $\cdot$ S. C. Serra $\cdot$ R. Portugal $\cdot$ F. G. Teixeira $\cdot$ \\ M. M. Carvalho $\cdot$ N. Silva $\cdot$ J. Espregueira-Mendes $\cdot$ \\ N. Sousa $\cdot$ A. J. Salgado
}

Received: 11 May 2014 / Accepted: 13 November 2014

(C) European Society of Sports Traumatology, Knee Surgery, Arthroscopy (ESSKA) 2014

\begin{abstract}
Purpose Massive rotator cuff tears (MRCT) are usually chronic lesions that present associated degenerative changes of the myotendinous unit that have been implicated in limitations for surgical repair. In order to develop effective therapies, it is important to establish animal models that mimic the hallmarks of the injury itself. Therefore, in the present work, we aimed to (1) optimize a rodent animal model of MRCT that closely reproduces the fatty infiltration of the cuff muscles seen in humans and (2) describe the effects of unilateral or bilateral lesion in terms of histology and behaviour.

Methods Massive tear was defined as two rotator cuff tendons-supraspinatus and infraspinatus-section. Twentyone Wistar rats were randomly assigned to four groups: bilateral lesion (five animals), right-sided unilateral lesion (five animals), left-sided unilateral lesion (five animals) and control (six animals). Behaviour was analyzed with open field and staircase test, 16 weeks after lesion. After that,
\end{abstract}

N. Sevivas $(\bowtie) \cdot$ S. C. Serra $\cdot$ F. G. Teixeira $\cdot$ M. M. Carvalho

N. Silva $\cdot$ J. Espregueira-Mendes $\cdot$ N. Sousa $\cdot$ A. J. Salgado

Life and Health Sciences Research Institute (ICVS), School

of Health Sciences, University of Minho, Campus de Gualtar,

4710-057 Braga, Portugal

e-mail: nunosevivas@gmail.com

N. Sevivas $\cdot$ S. C. Serra $\cdot$ F. G. Teixeira $\cdot$ M. M. Carvalho $\cdot$ N. Silva $\cdot$ J. Espregueira-Mendes $\cdot$ N. Sousa $\cdot$ A. J. Salgado ICVS/3B's - PT Government Associate Laboratory, Braga, Guimarães, Portugal

N. Sevivas

Orthopaedics Department, Hospital de Braga, Braga, Portugal

N. Sevivas $\cdot$ J. Espregueira-Mendes

Clínica Espregueira-Mendes, FIFA Medical Centre of Excellence,

Estádio do Dragão, Porto, Portugal animals were killed, and the supraspinatus and infraspinatus muscles were processed.

Results Histologic analysis revealed adipocytes, fatty infiltration and atrophy in the injured side with a greater consistency of these degenerative changes in the bilateral lesion group. Behaviour analysis revealed a significant functional impairment of the fine motor control of the forepaw analyzed in staircase test where the number of eaten pellets was significantly higher in sham animals (sham $=7 \pm 5.0$; left unilateral $=2.6 \pm 3.0$; right unilateral $=0 \pm 0$; and bilateral $=0 \pm 0, p<0.05$ ). A trend to reach a lower level of steps, in more injured animals, was also observed (sham animals $=3 \pm 1.6>$ left unilateral $=2 \pm 2.1>$ right unilateral $=0.8 \pm 1.3>$ bilateral $=0.8 \pm 1.1$ ).

Conclusions The present study has been able to establish an animal model that disclosed the hallmarks of MRCT. This can now be used as a valuable, cost-effective, preclinical instrument to assist in the development of advanced tissue engineered strategies. Moreover, this animal model

R. Portugal

Pathology Department, Centro Hospitalar São João, Porto,

Portugal

J. Espregueira-Mendes

3B's Research Group, Biomaterials, Biodegradables and Biomimetics, Department of Polymer, Engineering, University of Minho, AvePark, Zona Industrial da Gandra, S. Cláudio do Barco 4806-909 Caldas das Taipas, Guimarães, Portugal

J. Espregueira-Mendes

Headquarters of the European Institute of Excellence on Tissue Engineering and Regenerative Medicine, University of Minho, AvePark, Zona Industrial da Gandra, S. Cláudio do Barco 4806-909 Caldas das Taipas, Guimarães, Portugal 
overcomes some of the limitations of those that have been reported so far and thus represents a more reliable source for the assessment of future therapeutic strategies with potential clinical relevance.

Keywords Massive rotator cuff tear - Animal model . Fatty infiltration $\cdot$ Behaviour analysis

\section{Introduction}

Massive rotator cuff tears (MRCT) can cause debilitating symptoms including a painful and pseudoparalitic shoulder [20]. Several definitions of a MRCT have been proposed based in different criteria like the diameter of the tear $(\geq 5 \mathrm{~cm})[9,13]$, the area of the defect [45], tear pattern and the mobility of the tear margins [6], but the easiest and most widely used proposition is based on the number of tendons affected, with a minimum of two totally torn rotator cuff tendons defining the lesion [17, 20, 48, 50].

MRCT are usually chronic lesions associated with myotendinous retraction [36, 49, 50], atrophy and fatty infiltration of the muscles $[16,23]$. Such characteristics are important prognostic factors for the outcome of a rotator cuff repair $[17,22,23,25,30,35,46]$ because they are associated with loss of elasticity of the myotendinous unit, which adversely affect and, at times, hamper surgical treatment of the lesion [23, 24, 26].

In an attempt to improve surgical results, there is currently an increasing interest in developing new strategies to facilitate repair and thereby improve the healing process of MRCT injury [8, 15, 27]. However, the use of new therapeutic modalities must be tested, prior to use in humans, and the development of animal models and computer simulation have played a key role in achieving this aim [34]. The importance given to in vivo tests requires that the chosen model be appropriate, cost-effective and adequately mimics the pathology [7].

Different animal models have been described to study rotator's cuff pathology such as rabbit [18, 41], dog [42], sheep [10, 19], rat [3, 21, 28, 32, 39] and mouse [31]. The rat has important advantages, among which we highlight the similarity of the rotator cuff anatomy relative to the human being [3, 43], easiness of handling and low maintenance cost [28], which makes it a good candidate for the study of basic patho-anatomical mechanisms and therapeutic intervention results, in this pathology. However, we should underline that the load on the forelimb, like in other quadruped animals, is different from the human shoulder due to the human orthostatic position.

Initial attempts to develop a rat model of MRCT faced difficulties in mimicking the degenerative alterations associated to this condition, mostly due to detachment of only one tendon (supraspinatus) and the high rate of tendinous adherences associated $[3,21]$. This problem was overcome, at least partially, with a model in which there are at least two tendons detached [14, 28, 32] and therefore represents MRCT more faithfully. However, to increase the degree of fatty infiltration, some authors added a suprascapular nerve section to the two rotator cuff tendon detachment [28, 32].

Thus, the present study aimed at optimizing, validating and further characterizing, both histologically and functionally, a chronic MRCT model in rat that can mimic as close as possible what happens in the humans without a definitive neurologic injury. We hypothesized that a twotendon rotator cuff tear in the rat model would result in muscle degeneration and that the extension of degeneration would be related with the number of sides affected. We further aimed at determining whether functional impairment of fine motor control would be affected in a similar fashion. Overall, the animal model herein presented overcomes some of the limitations of those that have been reported so far and thus represents a more reliable source for the assessment of future therapeutic strategies with potential clinical relevance.

\section{Materials and methods}

\section{Rat animal model}

Twenty-one male Wistar rats (16 weeks), housed in light and temperature controlled rooms and fed on standard diet, were randomly assigned to the experimental groups. Lesion was defined as supraspinatus and infraspinatus tenotomy, to simulate MRCT [28, 32, 39], and the cut-off point for chronicity was established at 16 weeks post-injury based in previous reports $[14,28]$. The experimental control (Sham) was defined as a skin incision and detachment of the trapezius from the spine of the scapula, without any gesture carried out in the rotator cuff tendons. Four groups were established: (1) bilateral lesion $(n=5)$; (2) Unilateral right lesion $(n=5)$; (3) Unilateral left lesion $(n=5)$; and (4) Sham $(n=6)$.

\section{Surgical procedure}

All animals were anesthetized by intraperitoneal injection of a 1.5:1 mixture of ketamine $(60 \mathrm{mg} / \mathrm{ml}$, Imalgen, Merial, Portugal) and medetomidine hydrochloride $(0.4 \mathrm{mg} / \mathrm{ml}$, Dorbene Vet, Laboratorios Syva SA, Spain). Once anesthetized, fur was shaved in the area of the surgical site, the skin disinfected with chlorohexidine (AGB, Spain), and the animals were placed in prone position with the forelimbs in external rotation. A transverse incision was made in the skin and underlying fascia over the posterior aspect of the 
shoulder, followed by detachment of the trapezius from the spine of the scapula. The muscles of the rotator cuff were visualized with the supraspinatus (located in a superior position to the spine of the scapula) and the infraspinatus (inferior to the spine of the scapula) inserting into the greater tuberosity of the humerus. The acromion was carefully lifted to improve exposure of the area of insertion of the tendons that were then cut close to the bone and were allowed to retract medially (Fig. 1). A marking suture of Prolene 4-0 (Ethicon, Johnson and Johnson, USA) was placed on the end of the cut tendons to facilitate identification at the time of dissection. The trapezius muscle and the overlying skin were closed in successive layers with Vicryl 2-0 (Ethicon, Johnson and Johnson, USA). After surgery, analgesia (butorphanol tartrate, $1 \mathrm{mg} / \mathrm{ml}$, Fort Dodge, Spain) and antibiotic (enrofloxacin, $1 \mathrm{mg} / \mathrm{ml}$, Bayer, Germany) were administered, and the rats were kept under heat lamps until they were transferred to their cage, after which unrestricted activity was allowed.

\section{Motor behaviour analysis}

In order to understand the functional implication of the injury in an animal model and to analyze the efficacy of a given therapeutic intervention, it is also important to evaluate shoulder function [39]. Behavioural analysis in laboratory rodents is extensively used and validated in several contexts, namely in neurologic pathologies [44]. Some of these tests, especially those analyzing motor behaviour, including skilled limb movements, may be adequate to evaluate shoulder function. Behavioural open field [40, 44] and staircase [37] tests were performed at 16 weeks postinjury during the course of this work.

\section{Open field}

This test allows the assessment of motor behaviour through quantification of the rearing activity and the distance travelled, in a 5-min period. The rat is placed in the centre of a square arena $(43.2 \mathrm{~cm} \times 43.2 \mathrm{~cm})$ with transparent acrylic walls and in a brightly illuminated room (Med Associates Inc., St. Albans, VT, USA). The total distance travelled and the number of rearings are automatically registered by equipment sensors, evaluating the explorative behaviour of the animal in this environment.

\section{Staircase test}

This test evaluates fine motor control and positioning in the space of the forelimb. The test apparatus consists of a clear chamber with a hinged lid that was developed to assess the independent forelimb use in skilled reaching and grasping task. A narrow compartment, with a central raised platform

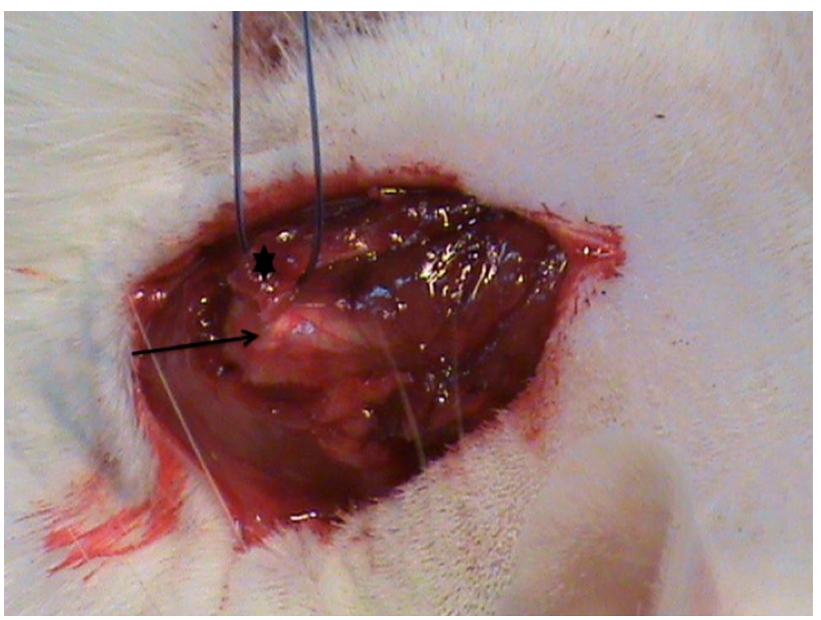

Fig. 1 Surgical procedure in the animal model. Star acromion lifted to improve exposure of the area of insertion of the tendons. Arrow supraspinatus and infraspinatus inserting on the greater tuberosity of the humerus

running along its length, is connected to this chamber. A removable double staircase, with seven steps on each side, is inserted in the space between the platform and the box walls. Each of the steps contains a small well, and three saccharin-flavoured pellets are placed in each well. The number of pellets eaten during the test period indicates the rat's success in grasping and retrieving the pellets. The number of steps from which pellets have been removed provides an index of the attempts to reach the food and how far the rat can reach.

Animals were pre-trained on the 4 days before the actual test and are food deprived during this time (animals are allowed to eat only $1 \mathrm{~h}$ per day). The protocol consisted of 2 days of testing during $5 \mathrm{~min}$ and then another consecutive 2 days of 10 min' test. On the 5th day, animals performed the test where the total amount of eaten pellets and the number of steps reached (where pellets were removed) were registered.

\section{Histology}

Sixteen weeks after the surgical procedure and after the behavioural analysis, the rats were deeply anesthetized by intra-peritoneal injection of sodium pentobarbital (Ceva Saude Animal, Portugal) and killed. The myotendinous units were harvested, embedded in optimum cutting temperature $(\mathrm{OCT})$ compound and stored at $-80{ }^{\circ} \mathrm{C}$. Frozen samples were cut with a cryostat in $20-\mu$ m-thick sections at the coronal plane and then placed on glass slides ( $24 \mathrm{sec}-$ tions for each muscle). Haematoxylin and Eosin (HE) and Oil Red O stains were performed to assess the presence of intra-muscular adipocytes, muscle atrophy and fatty infiltration [28]. 
In Oil Red O staining, lipids exhibit red colour, highlighting its presence. The amount of fat was evaluated with Oil Red $\mathrm{O}$ and graded semi-quantitatively on a four stage scale (1-4) in a manner based on that used by Goutallier for CT scan [23] except for the fact that we joined the stage 0 and 1 in a single stage 1 (stage 1 -absence to rare presence of fat deposits; stage 2-there is important fatty infiltration but there is still more muscle than fat; stage 3 -there is as much fat as muscle; and in stage 4, more fat than muscle is present and so we can find numerous fat deposits or droplets present in the majority of muscle fibres). The HE staining allowed a semi-quantitative assessment of the amount of intramuscular adipocytes, which was graded from 1 to 4 (grade 1 -absence to rare presence of adipocytes; grade 2-some groups of adipocytes; grade 3-the number of adipocytes are equivalent to the amount of muscle in at least one field of small magnification-40x; and grade 4-predominance of adipocytes in at least one field of small magnification). This staining was also used to assess muscle fibre atrophy that was graded
Fig. 2 Open field analysis. a Total distance; $\mathbf{b}$ vertical counts (bilateral $=5$; right unilateral $n=5$; left unilateral $=5$; sham $n=6$; mean $\pm \mathrm{SD}, p<0.05$ ) $361 \times 270 \mathrm{~mm}(72 \times 72 \mathrm{DPI})$
Fig. 3 Staircase test. a Total eaten pellets analysis; $\mathbf{b}$ right side eaten pellets analysis; $\mathbf{c}$ left side eaten pellets analysis; $\mathbf{d}$ level (number of steps) reached analysis. (bilateral $=5$; right unilateral $n=5$; left unilateral $=5$; $\operatorname{sham} n=6$; mean $\pm \mathrm{SD}, p<0.05)$ $361 \times 270 \mathrm{~mm}(72 \times 72 \mathrm{DPI})$
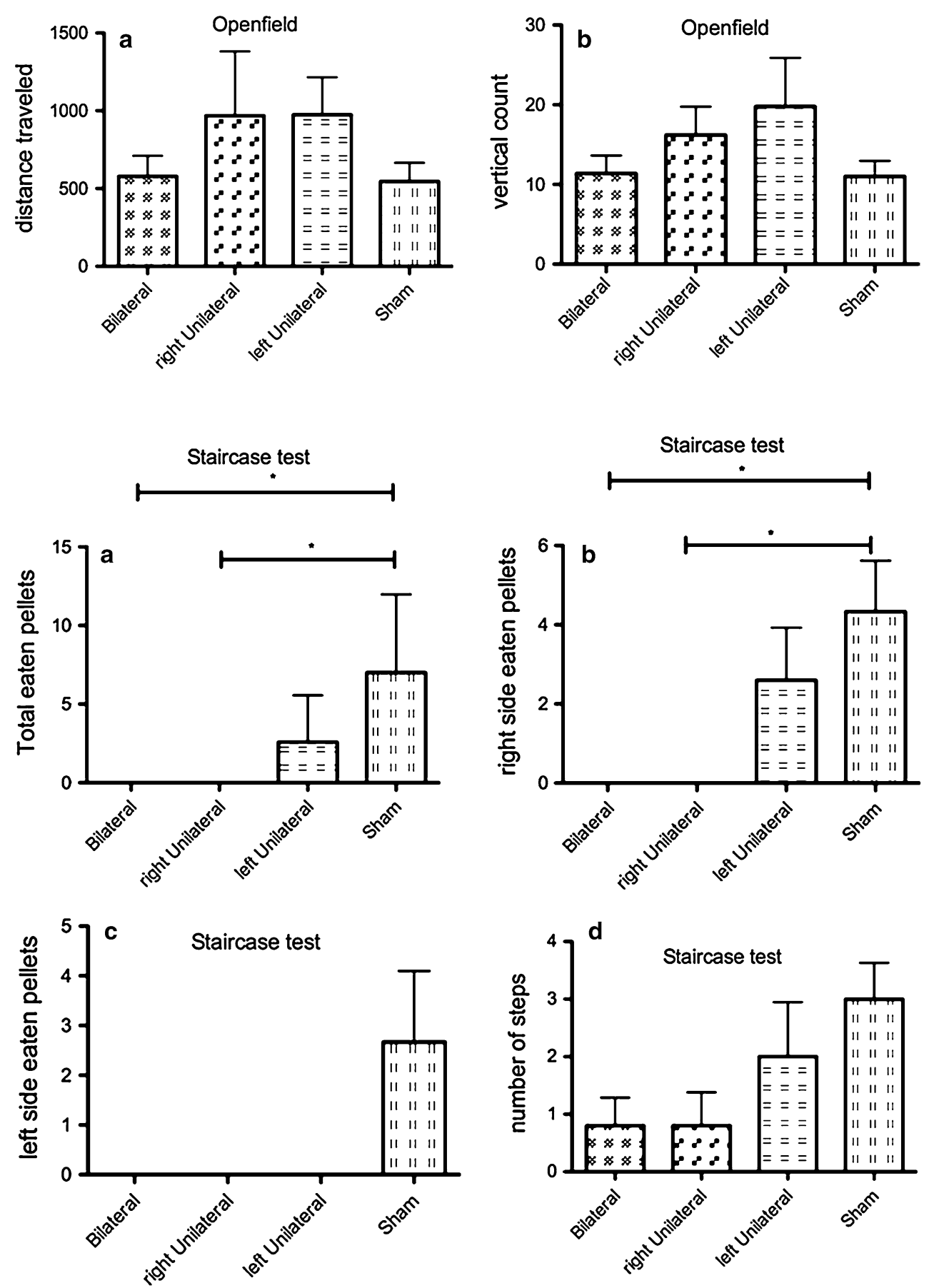
Table 1 Descriptive statistics of behaviour and histologic analysis (mean \pm SD)

\begin{tabular}{|c|c|c|c|c|}
\hline & Bilateral & Right unilateral & Left unilateral & Sham \\
\hline $\mathrm{OF}$-distance travalled & $578.2(294.7)$ & $968.3(923.9)$ & $974.2(539.3)$ & $545.3(294.5)$ \\
\hline OF-vertical counts & $11.4(5.0)$ & $16.2(7.9)$ & $19.8(13.6)$ & $11.0(4.8)$ \\
\hline Staircase_-total eaten pellets & $0.0(0.0)$ & $0.0(0.0)$ & $2.6(3.0)$ & $7.0(5.0)$ \\
\hline Staircase—right side eaten pellets & $0.0(0.0)$ & $0.0(0.0)$ & $2.6(3.0)$ & $4.3(3.1)$ \\
\hline Staircase_-left side eaten pellets & $0.0(0.0)$ & $0.0(0.0)$ & $0.0(0.0)$ & $2.7(3.5)$ \\
\hline Staircase_level reached & $0.8(1.1)$ & $0.8(1.3)$ & $2.0(2.1)$ & $3.0(1.6)$ \\
\hline Fatty infiltration—right SS muscle & $2.4(0.9)$ & $2.7(0.5)$ & $1.0(0.0)$ & $1.0(0.0)$ \\
\hline Fatty infiltration-left SS muscle & $3.2(0.8)$ & $1.2(0.4)$ & $2.2(0.8)$ & $1.0(0.0)$ \\
\hline Fatty infiltration-right IS muscle & $3.0(0.7)$ & $3.0(0.0)$ & $1.2(0.4)$ & $1.0(0.0)$ \\
\hline Fatty infiltration-left IS muscle & $2.6(0.9)$ & $1.2(0.4)$ & $2.4(0.6)$ & $1.0(0.0)$ \\
\hline Intramuscular adypocites_right SS muscle & $2.0(0.7)$ & $2.0(0.0)$ & $1.0(0.0)$ & $1.0(0.0)$ \\
\hline Intramuscular adypocites-left SS muscle & $2.8(0.4)$ & $1.0(0.0)$ & $1.8(0.4)$ & $1.0(0.0)$ \\
\hline Intramuscular adypocites_right IS muscle & $2.8(0.8)$ & $2.8(0.8)$ & $1.0(0.0)$ & $1.0(0.0)$ \\
\hline Intramuscular adypocites_-left IS muscle & $2.2(0.4)$ & $1.0(0.0)$ & $1.2(0.4)$ & $1.0(0.0)$ \\
\hline Atrophy_right SS muscle & $1.6(0.6)$ & $1.5(0.6)$ & $1.2(0.4)$ & $1.0(0.0)$ \\
\hline Atrophy—left SS muscle & $2.0(0.0)$ & $0.8(0.8)$ & $1.2(0.4)$ & $1.2(0.7)$ \\
\hline Atrophy—right IS muscle & $2.0(1.0)$ & $1.6(0.5)$ & $1.2(0.4)$ & $1.2(0.4)$ \\
\hline Atrophy_left IS muscle & $1.4(0.5)$ & $0.8(0.8)$ & $1.2(0.4)$ & $1.2(0.8)$ \\
\hline
\end{tabular}

Fig. 4 Oil Red O staining of supraspinatus muscle. a Fatty infiltration analysis in right supraspinatus muscle; b fatty infiltration analysis in left supraspinatus muscle; $\mathbf{c}$ stage 3 fatty infiltration (fat stained red) of the right supraspinatus muscle in a bilateral lesion animal (Oil Red $\mathrm{O} \times 100$ ); d stage 4 fatty infiltration of the left supraspinatus muscle in a bilateral lesion animal (Oil Red O $\times 40)$ (bilateral $=5$; right unilateral $n=5$; Left unilateral $=5$; sham $n=6$; mean $\pm \mathrm{SD}$, $p<0.05) 361 \times 270 \mathrm{~mm}$ $(72 \times 72$ DPI $)$
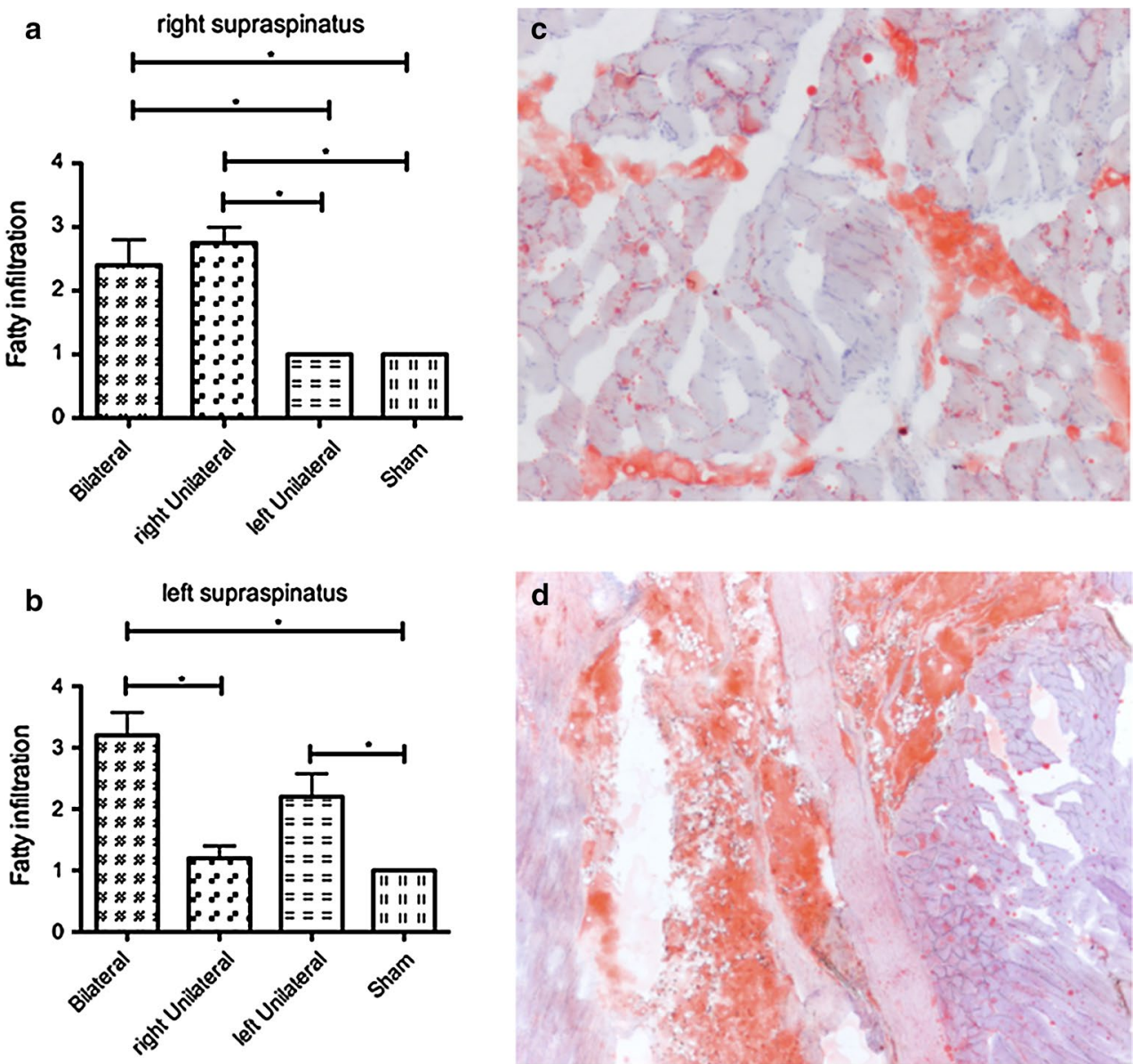
Fig. 5 HE staining of supraspinatus muscle. a Intramuscular adipocytes analysis in right supraspinatus muscle; b intramuscular adipocytes analysis in left supraspinatus muscle; c grade 3 intramuscular adipocytes of the right supraspinatus muscle of a bilateral lesion animal $(\mathrm{HE} \times 100)$; $\mathbf{d}$ grade 2 intramuscular adipocytes of the left supraspinatus muscle (lesion muscle) in an unilateral left lesion animal $(\mathrm{HE} \times 40)$ (bilateral $=5$; right unilateral $n=5$; left unilateral $=5$; sham $n=6$; mean $\pm \mathrm{SD}, p<0.05)$ $361 \times 270 \mathrm{~mm}(72 \times 72 \mathrm{DPI})$
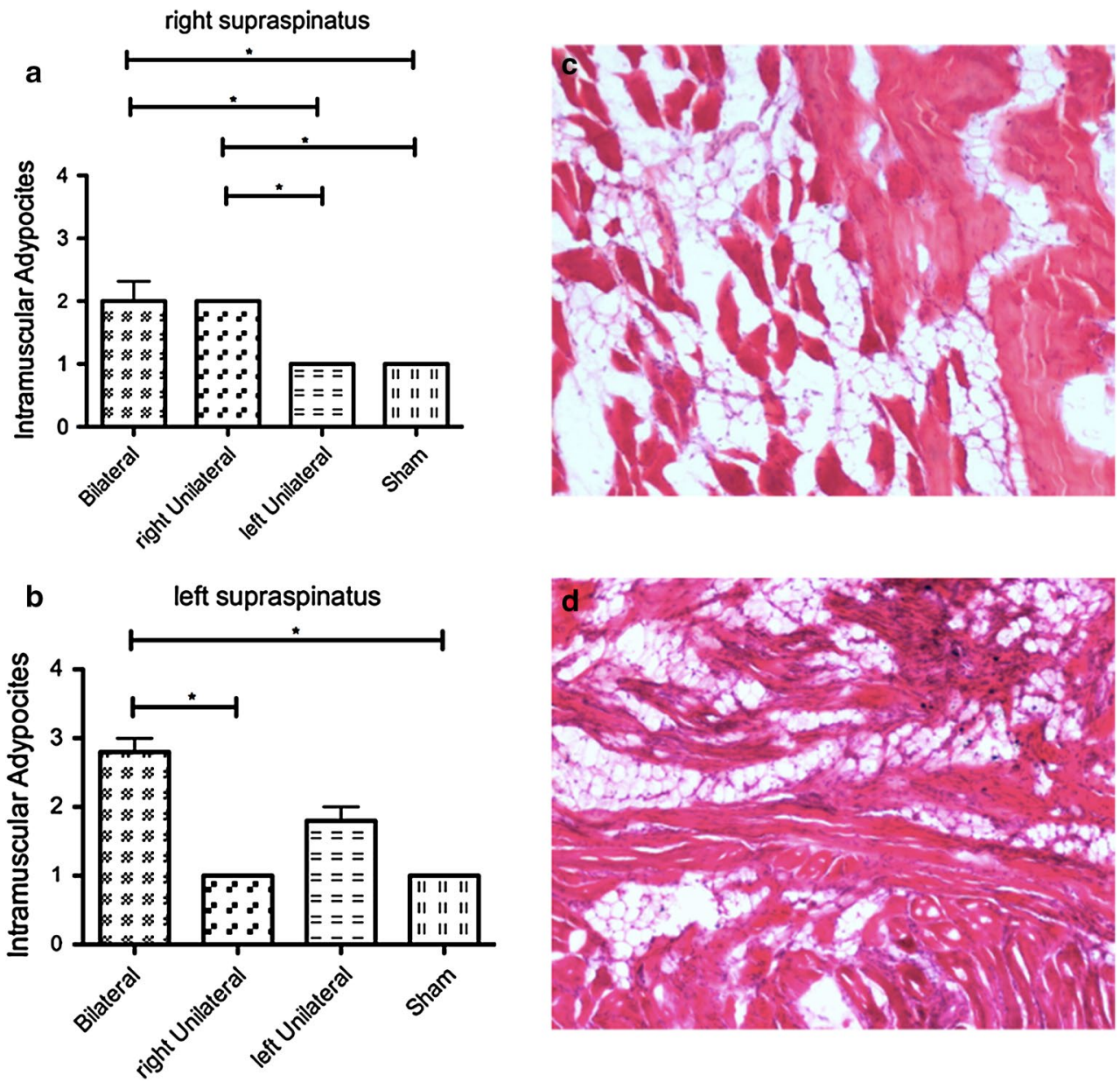

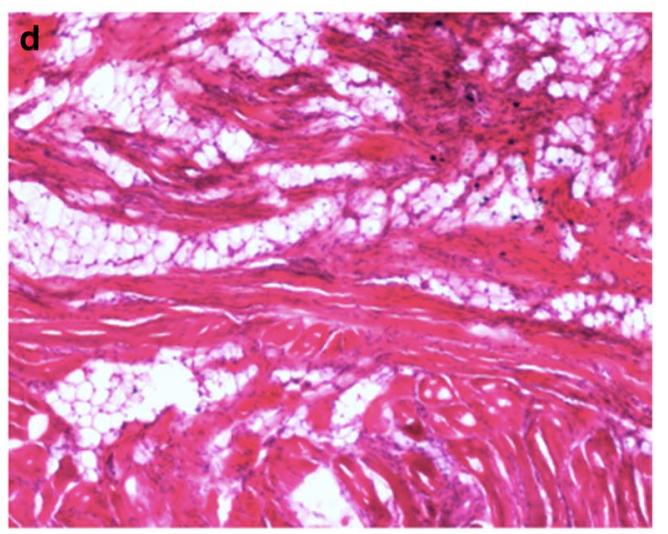

semi-quantitatively from 0 to 3 (grade 0 -no atrophy; grade 1-mild atrophy; grade 2-moderate atrophy; and grade 3-severe atrophy). This evaluation was based on aspects such as the apparent reduction in the size of muscle fibres and their angular shape. An independent pathologist conducted histological analysis, with the animal groups blinded.

The Animal Care Committee of the Life and Health Sciences Research Institute (ICVS)/School of Health Sciences of University of Minho approved the research protocols in accordance with standardized Animal Care Guidelines [47].

\section{Statistical analysis}

For Histology data, statistical analysis was performed using the nonparametric Kruskal-Wallis test to identify differences between groups, followed by the Dunn's multiple comparison test to assess which groups were significantly different. $p<0.05$ was used to indicate a statistically significant difference.

Behaviour statistical analysis was performed using a "one-way ANOVA" test followed by a Bonferroni post-test to evaluate statistical differences among groups. Statistical significance was defined for $p<0.05$. All data are presented as mean $\pm \mathrm{SD}$.

\section{Results}

Motor behavioural analysis

Open field analyses (Fig. 2) revealed that the total distance travelled in the arena, just after the surgery, was not significantly different between all groups (n.s.) (Fig. 2a). The number of rearing events (exploratory behaviour where animals stand up only in their hind paws) was also assessed, and once again animals from all groups had a similar performance without significant differences (n.s.) (Fig. 2b). This data probably indicates that the chronic lesion of the rotator cuff does not affect the locomotion ability or the rearing behaviour of the rat because these activities are more dependent on the hind limb function.

The staircase test (Fig. 3) was performed at 16 weeks post-injury to assess fine motor control of the forelimb, as this function should be impaired with a chronic and massive tear of the rotator cuff. After a 4-day training period, 
Fig. 6 Muscular atrophy analysis. a Muscular atrophy analysis in right supraspinatus muscle; b muscular atrophy analysis in left supraspinatus muscle; c muscular atrophy analysis in right infraspinatus muscle; d muscular atrophy analysis in left infraspinatus muscle (bilateral $=5$; right unilateral $n=5$; left unilateral $=5$; sham $n=6$; mean $\pm \mathrm{SD}, p<0.05)$ $361 \times 270 \mathrm{~mm}(72 \times 72 \mathrm{DPI})$
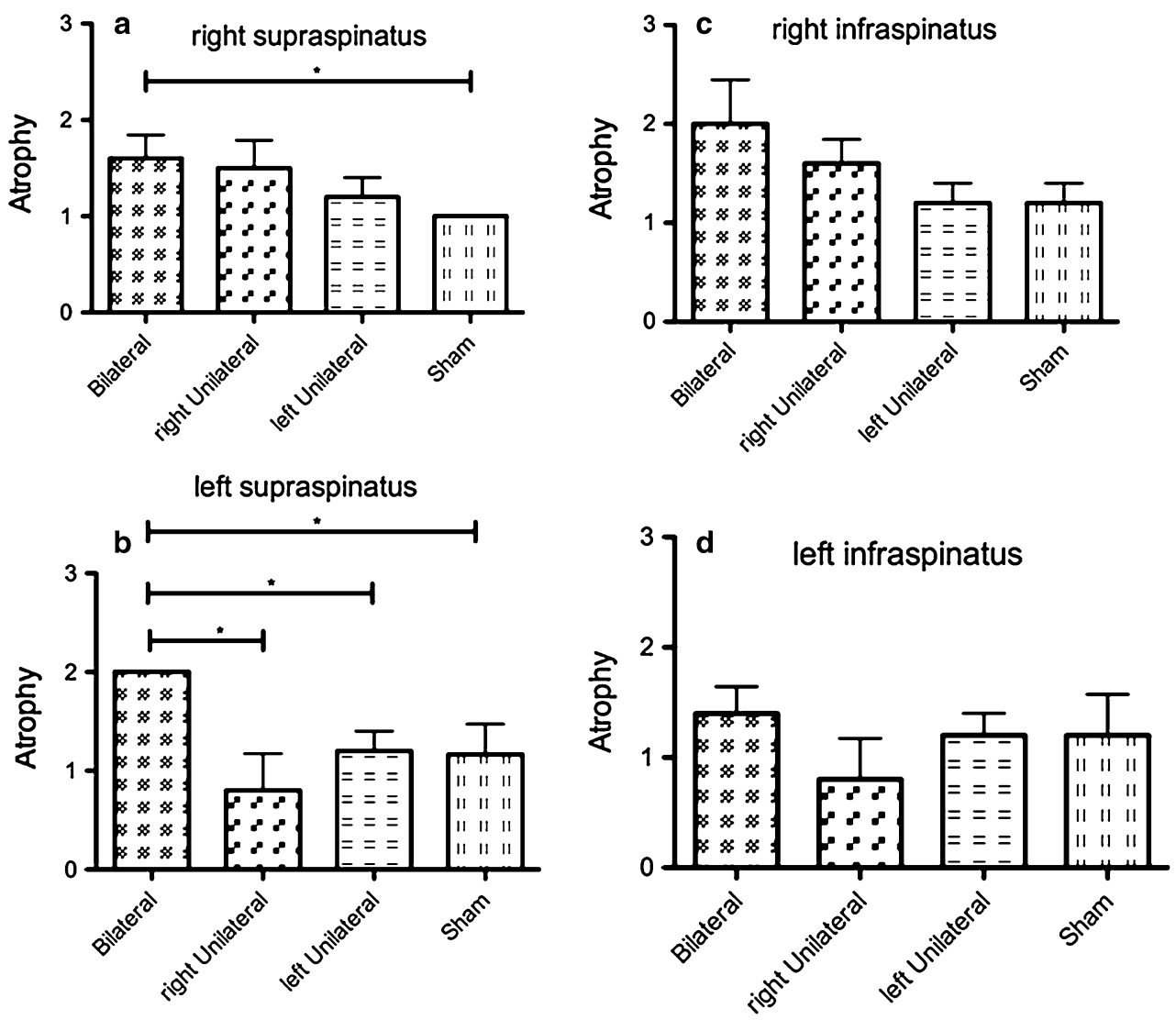

the test was undertaken, and the number of eaten pellets was significantly higher $(p<0.05)$ in sham animals (Fig. 3a). When we analyze right (Fig. 3b) and left (Fig. 3c) sides independently, we only found significant differences in the right side $(p<0.05)$ between sham animals compared with bilateral and right unilateral lesion (Fig. 3b).

In the number of steps reached, where the pellets were withdrawn (eaten or pulled out), we observed a smaller number in ascending order on bilateral $(0.8 \pm 1.1)<$ right unilateral $(0.8 \pm 1.3)<$ left unilateral $(2 \pm 2.1)<$ sham animals $(3 \pm 1.6)$. This reflects a trend to reach a higher level of steps if the rat has "less lesion"; however, we did not find statistically significant differences (n.s.) (Fig. 3d).

Descriptive statistics is summarized in Table 1.

Histologic analysis

The results of fatty infiltration (Oil Red O), the amount of intramuscular adipocytes (HE) and muscular atrophy (HE), are presented separately and complemented with images and graphs to facilitate the comprehension of the data. Figures 4, 5, 6, 7 and 8 reveal the data obtained for these parameters in the supraspinatus and infraspinatus muscles.

In the right supraspinatus and infraspinatus muscles, there was a significant increase $(p<0.05)$ in fatty infiltration in animals with bilateral and right unilateral lesion compared with left unilateral and sham animals. Additionally, it was also found a significantly increased number of adipocytes $(p<0.05)$ in the same groups. The degree of atrophy, in these same muscles, was greater in animals with bilateral and right unilateral lesion. This was more evident for the right supraspinatus, where significant differences $(p<0.05)$ were obtained between the bilateral lesion group relative to the sham group.

In the left supraspinatus and infraspinatus muscles, there was a significant increase of fatty infiltration in animals with bilateral and left unilateral lesion $(p<0.05)$. Moreover, the bilateral group also disclosed a higher rate of atrophy and infiltration of adipocytes.

\section{Discussion}

The most important findings of the present study were that injuring the upper limb of a rat through the tenotomy of supraspinatus and infraspinatus leads to: (1) atrophy and fatty degeneration of the muscle; and (2) an impairment of fine motor skills dependent on the shoulder function, similar to what is seen clinically. Both of these observations were more noticeable when both paws are injured. 
Fig. 7 Oil Red O staining of infraspinatus muscle. a Fatty infiltration analysis in right infraspinatus muscle; b fatty infiltration analysis in left infraspinatus muscle; c stage 4 fatty infiltration of the right infraspinatus muscle of a bilateral lesion animal (Oil Red O $\times 40$ ); $\mathbf{d}$ stage 1 fatty infiltration of the right infraspinatus muscle in a sham animal (Oil Red $\mathrm{O} \times 40)$ (bilateral $=5$; right unilateral $n=5$; left unilateral $=5$; sham $n=6$; mean $\pm \mathrm{SD}$, $p<0.05) 361 \times 270 \mathrm{~mm}$ $(72 \times 72$ DPI $)$
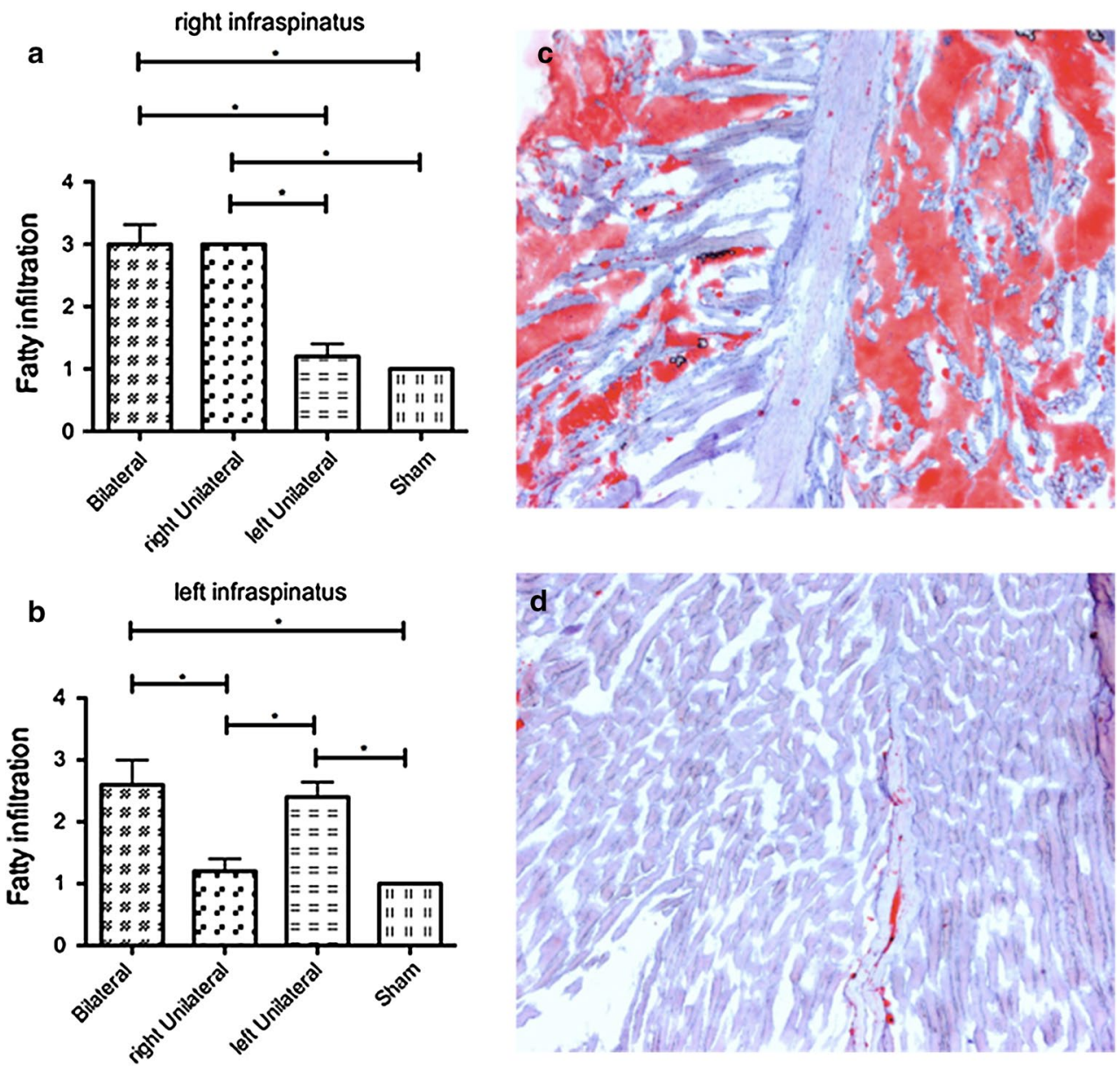

Some important points to consider when developing an animal model are: (1) the anatomical structure used in the model should be representative of the injured structure; and (2) the pathological condition must be simulated as accurately as possible (e.g. partial $v s$ complete rupture, small $v s$ large $v s$ massive tears, neuropraxia vs neurotmesis) because different conditions require different models. In another dimension, it is critical to have tools that accurately evaluate the effectiveness of the model, either histologically and from the functional point of view [7].

Two previous studies using the rat model [28, 32] showed that the suprascapular nerve section, in association with a two rotator cuff tendon detachment, significantly increases the degree of fatty infiltration and muscular atrophy. However, in humans, the suprascapular nerve injury, which can be associated with MRCT, is a traction injury (neuropraxia) and has the potential for recovery after tendon repair $[1,2,5,12,33]$. As the neurologic injury in those animal models is a definitive section (neurotmesis), it represents a different type of lesion and does not represent faithfully what happens in MRCT.

The muscles on the injured side presented degenerative changes, and a statistically significant difference was found relative to sham and non-injured side in the model. However, it appears that there is a greater constancy and homogeneity in the degenerative changes associated with the bilateral lesion group, and there is a trend for more marked changes than in the unilateral lesion group (although only rarely these differences reach statistical significance).

Therefore, the results indicate that degenerative changes may be aggravated in order to represent in a more accurate way the changes observed in humans, with the use of an animal model of bilateral lesion. This may be due to a greater immobility of the animal after bilateral lesion, leading to a higher degree of fatty infiltration and muscular atrophy.

This is, to the best of our knowledge, the first time that such a model is described, offering the additional advantage of allowing using the two sides of the same animal, to assess different therapeutic interventions, reducing the number of animals required to conduct experiments, thus being ethically very defensible. The development of this rat model of MRCT should now proceed to investigate a reproducible functional assessment and evaluation of the associated pain. This would help to reveal the functional significance of the lesion and the outcome of therapeutic 
Fig. 8 HE staining of infraspinatus muscle. a Intramuscular adipocytes analysis in right infraspinatus muscle; b intramuscular adipocytes analysis in left infraspinatus muscle; $\mathbf{c}$ grade 4 intramuscular adipocytes of the right infraspinatus muscle in a bilateral lesion animal $(\mathrm{HE} \times 100)$; $\mathbf{d}$ grade 1 intramuscular adipocytes of the left infraspinatus muscle in a sham animal $(\mathrm{HE} \times 40)$ (bilateral $=5$; right unilateral $n=5$; left unilateral $=5$; sham $n=6 ;$ mean $\pm \mathrm{SD}, p<0.05)$ $361 \times 270 \mathrm{~mm}(72 \times 72 \mathrm{DPI})$

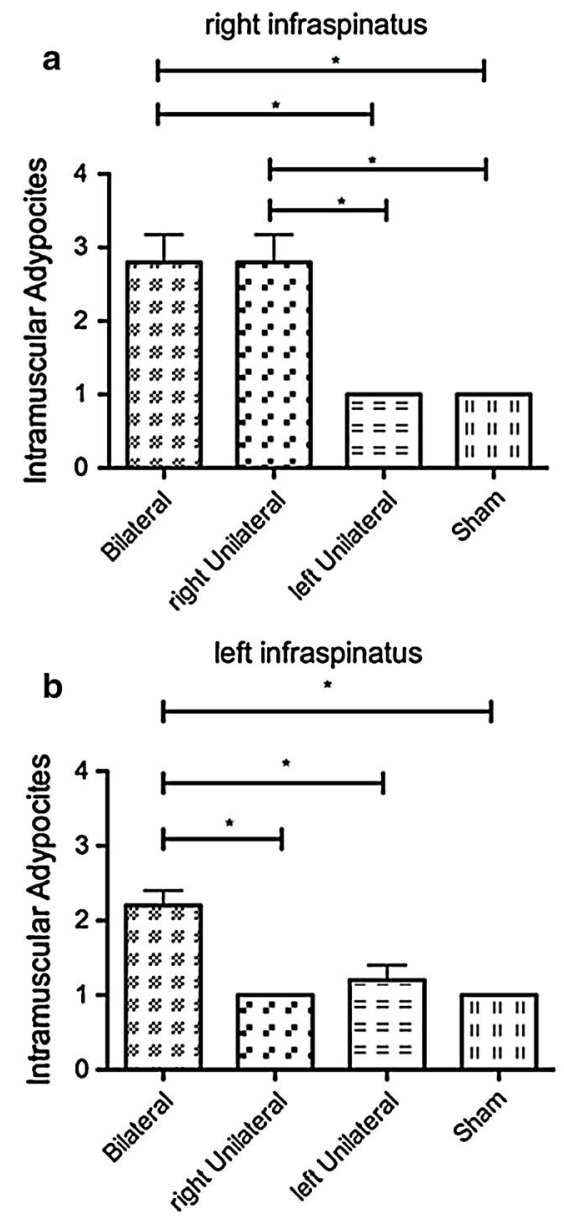

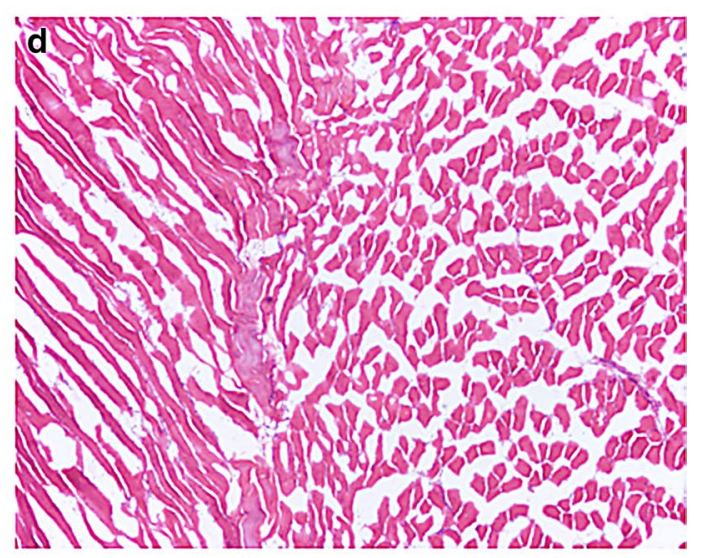

interventions, from a functional standpoint, prior to the killing of the animal. A functional index with evaluation of movement, strength and pain, like the one used in clinical practice [11] or in other anatomical locations in the same animal model [38], would be an ideal tool and may be used also to assess a possible correlation with the histological data.

There are some limitations that can be identified in this study: (1) the reduced number of animals enrolled limits the power of the analyses; (2) the semi-quantitative assessment of histological features is not a very precise assessment, even though it is similar to the method used in clinical practice; (3) the injury inflicted is an acute injury, in an young rat, that affects previously healthy tendon, as opposed to what commonly occurs in humans, where there are prior degenerative changes; (4) apoptosis is increased in rotator cuff pathology and plays a key role in the development of the characteristic degenerative changes [4, 29]. However, this is not the case in this model, and the possible activation and increased activity of this homeostatic process, after the acute injury, were not studied; and (5) the single time point of all the presented analyses, and it would be interesting to investigate the kinetics of histology and behaviour following chronic lesion in more time points.

As a consequence of what was discussed above, the animal model presented in this report can represent a reliable source for the assessment of future therapeutic strategies.

\section{Conclusion}

The rat model developed in this study shows histological features that are similar to those seen clinically, especially in the bilateral injury model. This should pave the way for further studies leading to a better understanding of the molecular mechanisms underlying the chronic degenerative changes of the myotendinous unit, development and testing of new therapeutic strategies aiming at preventing or reverting these same changes and enhance the potential healing of these tissues.

Acknowledgments Portuguese Foundation for Science and Technology (FCT) (FCT Development Grant to A.J. Salgado). This work was also co-funded by Programa Operacional Regional do Norte (ON.2-O Novo Norte), ao abrigo do Quadro de Referência Estratégico Nacional (QREN), através do Fundo Europeu de Desenvolvimento Regional (FEDER). 
Conflict of interest The authors declare that they have no conflict of interest.

\section{References}

1. Albritton MJ, Graham RD, Richards RS 2nd, Basamania CJ (2003) An anatomic study of the effects on the suprascapular nerve due to retraction of the supraspinatus muscle after a rotator cuff tear. J Shoulder Elbow Surg 12(5):497-500

2. Asami A, Sonohata M, Morisawa K (2000) Bilateral suprascapular nerve entrapment syndrome associated with rotator cuff tear. $\mathbf{J}$ Shoulder Elbow Surg 9(1):70-72

3. Barton ER, Gimbel JA, Williams GR, Soslowsky LJ (2005) Rat supraspinatus muscle atrophy after tendon detachment. J Orthop Res 23(2):259-265

4. Benson RT, McDonnell SM, Knowles HJ, Rees JL, Carr AJ, Hulley PA (2010) Tendinopathy and tears of the rotator cuff are associated with hypoxia and apoptosis. J Bone Joint Surg $\mathrm{Br}$ 92(3):448-453

5. Boykin RE, Friedman DJ, Higgins LD, Warner JJ (2010) Suprascapular neuropathy. J Bone Joint Surg Am 92(13):2348-2364

6. Burkhart SS (1991) Arthroscopic treatment of massive rotator cuff tears. Clinical results and biomechanical rationale. Clin Orthop Relat Res 267:45-56

7. Carpenter JE, Hankenson KD (2004) Animal models of tendon and ligament injuries for tissue engineering applications. Biomaterials 25(9): 1715-1722

8. Chang CH, Chen CH, Su CY, Liu HT, Yu CM (2009) Rotator cuff repair with periosteum for enhancing tendon-bone healing: a biomechanical and histological study in rabbits. Knee Surg Sports Traumatol Arthrosc 17(12):1447-1453

9. Cofield RH, Parvizi J, Hoffmeyer PJ, Lanzer WL, Ilstrup DM, Rowland CM (2001) Surgical repair of chronic rotator cuff tears. A prospective long-term study. J Bone Joint Surg Am 83-A(1):71-77

10. Coleman SH, Fealy S, Ehteshami JR, MacGillivray JD, Altchek DW, Warren RF, Turner AS (2003) Chronic rotator cuff injury and repair model in sheep. J Bone Joint Surg Am 85-A(12):2391-2402

11. Constant CR, Murley AH (1987) A clinical method of functional assessment of the shoulder. Clin Orthop Relat Res 214:160-164

12. Costouros JG, Porramatikul M, Lie DT, Warner JJ (2007) Reversal of suprascapular neuropathy following arthroscopic repair of massive supraspinatus and infraspinatus rotator cuff tears. Arthroscopy 23(11):1152-1161

13. DeOrio JK, Cofield RH (1984) Results of a second attempt at surgical repair of a failed initial rotator-cuff repair. J Bone Joint Surg Am 66(4):563-567

14. Dourte LM, Perry SM, Getz CL, Soslowsky LJ (2010) Tendon properties remain altered in a chronic rat rotator cuff model. Clin Orthop Relat Res 468(6):1485-1492

15. Ellera Gomes JL, da Silva RC, Silla LM, Abreu MR, Pellanda R (2012) Conventional rotator cuff repair complemented by the aid of mononuclear autologous stem cells. Knee Surg Sports Traumatol Arthrosc 20(2):373-377

16. Fuchs B, Weishaupt D, Zanetti M, Hodler J, Gerber C (1999) Fatty degeneration of the muscles of the rotator cuff: assessment by computed tomography versus magnetic resonance imaging. $\mathbf{J}$ Shoulder Elbow Surg 8(6):599-605

17. Gerber C, Fuchs B, Hodler J (2000) The results of repair of massive tears of the rotator cuff. J Bone Joint Surg Am 82(4):505-515

18. Gerber CMD, Nuss KM, Farshad M (2011) Anabolic steroids reduce muscle damage caused by rotator cuff tendon release in an experimental study in rabbits. J Bone Joint Surg Am 93(23):2189-2195
19. Gerber C, Meyer DC, Schneeberger AG, Hoppeler H, von Rechenberg B (2004) Effect of tendon release and delayed repair on the structure of the muscles of the rotator cuff: an experimental study in sheep. J Bone Joint Surg Am 86(9):1973-1982

20. Gerber C, Wirth SH, Farshad M (2011) Treatment options for massive rotator cuff tears. J Shoulder Elbow Surg 20(2 Suppl):S20-S29

21. Gimbel JA, Van Kleunen JP, Mehta S, Perry SM, Williams GR, Soslowsky LJ (2004) Supraspinatus tendon organizational and mechanical properties in a chronic rotator cuff tear animal model. J Biomech 37(5):739-749

22. Gladstone JN, Bishop JY, Lo IK, Flatow EL (2007) Fatty infiltration and atrophy of the rotator cuff do not improve after rotator cuff repair and correlate with poor functional outcome. Am J Sports Med 35(5):719-728

23. Goutallier D, Postel JM, Bernageau J, Lavau L, Voisin MC (1994) Fatty muscle degeneration in cuff ruptures. Pre- and postoperative evaluation by CT scan. Clin Orthop Relat Res 304:78-83

24. Goutallier D, Postel JM, Gleyze P, Leguilloux P, Van Driessche S (2003) Influence of cuff muscle fatty degeneration on anatomic and functional outcomes after simple suture of full-thickness tears. J Shoulder Elbow Surg 12(6):550-554

25. Goutallier D, Postel JM, Lavau L, Bernageau J (1998) Influence of muscular degeneration of the supra- and infra-spinatus on the prognosis of surgical repair of the rotator cuff. Acta Orthop Belg 64(Suppl 2):42-45

26. Goutallier D, Postel JM, Lavau L, Bernageau J (1999) Impact of fatty degeneration of the supraspinatus and infraspinatus muscles on the prognosis of surgical repair of the rotator cuff. Rev Chir Orthop Reparatrice Appar Mot 85(7):668-676

27. Gulotta LV, Kovacevic D, Packer JD, Deng XH, Rodeo SA (2011) Bone marrow-derived mesenchymal stem cells transduced with scleraxis improve rotator cuff healing in a rat model. Am J Sports Med 39(6):1282-1289

28. Kim HM, Galatz LM, Lim C, Havlioglu N, Thomopoulos S (2012) The effect of tear size and nerve injury on rotator cuff muscle fatty degeneration in a rodent animal model. J Shoulder Elbow Surg 21(7):847-858

29. Lee HJ, Kim YS, Ok JH, Song HJ (2013) Apoptosis occurs throughout the diseased rotator cuff. Am J Sports Med 41(10):2249-2255

30. Liem D, Lichtenberg S, Magosch P, Habermeyer P (2007) Magnetic resonance imaging of arthroscopic supraspinatus tendon repair. J Bone Joint Surg Am 89(8):1770-1776

31. Liu X, Laron D, Natsuhara K, Manzano G, Kim HT, Feeley BT (2012) A mouse model of massive rotator cuff tears. J Bone Joint Surg Am 94(7):e41

32. Liu X, Manzano G, Kim HT, Feeley BT (2011) A rat model of massive rotator cuff tears. J Orthop Res 29(4):588-595

33. Mallon WJ, Wilson RJ, Basamania CJ (2006) The association of suprascapular neuropathy with massive rotator cuff tears: a preliminary report. J Shoulder Elbow Surg 15(4):395-398

34. Mannava S, Plate JF, Tuohy CJ, Seyler TM, Whitlock PW, Curl WW, Smith TL, Saul KR (2013) The science of rotator cuff tears: translating animal models to clinical recommendations using simulation analysis. Knee Surg Sports Traumatol Arthrosc 21(7):1610-1619

35. Mellado JM, Calmet J, Olona M, Esteve C, Camins A, Perez Del Palomar L, Gine J, Sauri A (2005) Surgically repaired massive rotator cuff tears: MRI of tendon integrity, muscle fatty degeneration, and muscle atrophy correlated with intraoperative and clinical findings. AJR Am J Roentgenol 184(5):1456-1463

36. Meyer DC, Lajtai G, von Rechenberg B, Pfirrmann CW, Gerber C (2006) Tendon retracts more than muscle in experimental chronic tears of the rotator cuff. J Bone Joint Surg Br 88(11):1533-1538

37. Montoya CP, Campbell-Hope LJ, Pemberton KD, Dunnett SB (1991) The "staircase test": a measure of independent forelimb 
reaching and grasping abilities in rats. J Neurosci Methods 36(2-3):219-228

38. Murrell GA, Lilly EG, Davies H, Best TM, Goldner RD, Seaber AV (1992) The Achilles functional index. J Orthop Res 10(3):398-404

39. Perry SM, Getz CL, Soslowsky LJ (2009) Alterations in function after rotator cuff tears in an animal model. J Shoulder Elbow Surg 18(2):296-304

40. Prut L, Belzung C (2003) The open field as a paradigm to measure the effects of drugs on anxiety-like behaviors: a review. Eur J Pharmacol 463(1-3):3-33

41. Rubino LJ, Stills HF Jr, Sprott DC, Crosby LA (2007) Fatty infiltration of the torn rotator cuff worsens over time in a rabbit model. Arthroscopy 23(7):717-722

42. Safran O, Derwin KA, Powell K, Iannotti JP (2005) Changes in rotator cuff muscle volume, fat content, and passive mechanics after chronic detachment in a canine model. J Bone Joint Surg Am 87(12):2662-2670

43. Soslowsky LJ, Carpenter JE, DeBano CM, Banerji I, Moalli MR (1996) Development and use of an animal model for investigations on rotator cuff disease. J Shoulder Elbow Surg 5(5):383-392

44. Sousa N, Almeida OF, Wotjak CT (2006) A hitchhiker's guide to behavioral analysis in laboratory rodents. Genes Brain Behav 5(Suppl 2):5-24
45. Tauro JC (2006) Stiffness and rotator cuff tears: incidence, arthroscopic findings, and treatment results. Arthroscopy 22(6):581-586

46. Thomazeau H, Rolland Y, Lucas C, Duval JM, Langlais F (1996) Atrophy of the supraspinatus belly. Assessment by MRI in 55 patients with rotator cuff pathology. Acta Orthop Scand 67(3):264-268

47. Van Zutphen LFM, Baumans V, Beynen A (2001) Principles of laboratory animal science. Elsevier, Amsterdam

48. Warner JP, Krushell RJ, Masquelet A, Gerber C (1992) Anatomy and relationships of the suprascapular nerve: anatomical constraints to mobilization of the supraspinatus and infraspinatus muscles in the management of massive rotator-cuff tears. J Bone Joint Surg Am 74(1):36-45

49. Zingg PO, Jost B, Sukthankar A, Buhler M, Pfirmann CW, Gerber $C$ (2007) Clinical and structural outcomes of nonoperative management of massive rotator cuff tears. J Bone Joint Surg Am 89(9):1928-1934

50. Zumstein MA, Jost B, Hempel J, Hodler J, Gerber C (2008) The clinical and structural long-term results of open repair of massive tears of the rotator cuff. J Bone Joint Surg Am 90(11):2423-2431 